Abdel-Malek, K., Zou, H.L., Wang, J.Y., and Othman, S., (1999), "Automated Design and Parametrization of Mechanical Part geometry", Research in Engineering Design,

Vol. 11, pp. 206-217.

\title{
Automated Design and Parametrization of Mechanical Part Geometry
}

\author{
K Abdel-Malek ${ }^{1}$, H-L Zou ${ }^{2}$, and J-Y Wang ${ }^{2}$, and S Othman ${ }^{3}$ \\ ${ }^{1}$ Department of Mechanical Engineering and Center for Computer Aided Design (CCAD), The University of Iowa, \\ Iowa City, IA, USA; ${ }^{2}$ Structural Dynamics Research Corporation (SDRC), Research and Development \\ Department, Milford, OH, USA; ${ }^{3}$ US National Advanced Driving Simulator (NADS), Oakdale, IA, USA
}

Variational methods for evaluating the design of mechanisms were first introduced by this group in the form of mathematical formulations generally applicable to open- and closed-loop mechanisms. This method is herein extended and demonstrated to the design of mechanical parts in the context of automatic parametrization of the geometry. The formulation is based on the development of constraint equations that govern the relation between geometry in a mechanical part as dictated by a designer. Instead of the tedious method of specifying mathematical relations between any two geometries of the part, it is proposed to use the notion of kinematic relations inherent in the formulation relating the connectivity between joints and links. Cut-joint constraints are introduced, kinematic joints in the formulation are combined, their variations evaluated, and a Jacobian is determined. Constraint violations are then compensated to compute an assembled mechanism, hence redesigning the part. It is shown that this kinematically-driven formulation is broadly applicable to 2D and 3D models. The method and algorithm are illustrated through a number of examples.

Keywords: Cut-joint kinematic constraints, automatic computer-aided design, design propagation, automatic parametrization, constraint management.

\section{INTRODUCTION}

Automated design methods in computer aided design pertaining to parametrization and constraint definitions have met with great success in recent years. Commercial Computer Aided Design (CAD) computer code has provided versatile venues for end-users to automate the design process. Despite recent advances, the sophistication of these formulations remains at an early stage of development. The goal of this work is to introduce a new numerical method for automated parametrization of mechanical part geometry using the inherent kinematic properties of mechanisms. As a result, a method for automated CAD is developed.

Mechanical parts in an assembly modeled in a CAD system undergo many changes before reaching the optimal design state. The field of computer-aided design has seen a revolutionary advancement by the advent of parametric technology. Using this technology, many commercial CAD systems have simplified the iterative design process. In most cases, parametric technology has been implemented characterized by sets of rules associating dimensions by simple equations. For example, the length of two links $d_{1}$ and $d_{2}$ may be 
related parametrically by one equation as $d_{1}=d_{2} t$, where $t$ is the parameter. This indicates that a change in the length of link 2 will automatically change the length of link 1. Similarly, a change in any geometry causes a change in another geometric property. The goal is to automate the product development process within the mechanical CAD environment.

Computer code that implements the iterative concept of design and that automatically alters designs is reported by many researchers (Imamura 1994, Burke et al. 1994, and Cutkosky and Tenenbaum 1990). This type of software facilitates constraint-based modeling and in many cases provides inferencing via constraint propagation. Some methods specify a parametric dependency between different parts. A design change in the geometry of a part propagates through the complete design. Other efforts at CAD/CAM automation include reasoning schemes that use topological relationships between features (McMahon et al. 1997).

Examples of software that has recently appeared to support automated mechanical design include that developed by Bowen and Bahler (1992) using constraint networks, Fohn, et al. (1994) using constraint systems shells, and Kolb and Bailey (1993) using a constraint-based object modeling method. Other methods that have dealt with constraint management were addressed by Serrano and Gossard (1988). Works addressing this subject are numerous and cover a variety of disciplines including Aritificial Intelligence, Computer Aided Design, and Manufacturing. An important contribution to this field was made by Kott et al. (1992) where a configuration technique that is well suited for configuring 'decomposable' artifacts of a mechanical part with reasonably well defined structure and constraints was presented and demonstrated for an automotive component. Algorithms that provide the user with flexibility in choice of design specifications of constraint management were introduced by Agrawal et al. (1993), where the code was demonstrated for a number of mechanical parts.

Other programs dealing with constraint management and structural decomposition include the program called MEET (Steinberg and Lanagarana 1996). A method applied to the manufacturing industry using feature-based generative design-by-constraints was applied by Jaques et al. (1991), in which constraints are manipulated. More recent efforts to automate the design process in manufacturing were demonstrated by Abdel-Malek and Maropis ( 1998).

In this paper, design propagations due to a change in geometric parameters are considered throughout the mechanical part. The derivation of basic constraints in the context of automatic computer aided design was first introduced by Zou et al. (1996). It is based on a computational method for studying the kinematics of mechanical systems as first introduced in by Haug (1989). Basic constraints were first developed for the study of variational propagations in mechanism assembly design (Zou et al. 1998). In this paper, we generalize the formulation, present a robust method for computing a solution using the modified moore-penrose pseudo inverse, and demonstrate the work in the context of an automated CAD interface. Indeed, several spatial examples are treated and illustrated. The emphasis on masking this program (in an automated mode) from the CAD user is also addressed. Limitations of this work that are currently under development are also addressed.

It was shown earlier that constraints are derived that represent the connection between any two links and were called basic constraints (Zou et al. 1996). A cut-joint kinematic constraint formulation used in multibody dynamic analysis was implemented in representing the geometry 
of parts. Two cases were delineated and a simple example was demonstrated. It was shown that a mechanical part can be modeled as a simple mechanism comprising joints and links. A dimension was replaced by a specific link, while a change in length was modeled as a slidercrank pair. A change in the mechanical part was propagated through the mechanism and a new configuration was computed. The extension of this work to the automatic parametrization of 3D mechanical part geometry and the combination of joints in the analysis are presented in this paper. Combination of joints in the graph topology analysis leads to a simplified calculation (and faster computational time). The analysis in this paper is illustrated for three-dimensional geometrical parts that are converted to spatial mechanisms with a multiple of kinematic pairs.

General conventions used throughout the development of the method are first introduced in Section 2. Graph tree representation will be used to model the system using a spanning tree and a cut-joint constraint. A set of equations will be developed while maintaining vector quantities representing link length and orientation as variables, namely the Euler angles and the body-position vector (Section 3). These equations will then be linearized but having a nonsquare Jacobian. The Moore-Penrose pseudo inverse will be used to compute an assembled configuration using the kinematically driven formulation, hence automatically redesigning the mechanism subject to the specified change (Section 4). The new mechanism will then be converted back to a mechanical part, consequently providing a new design. Three examples will be illustrated in the context of automated computer-aided engineering for parametrization of geometric constraints.

\section{CONVENTIONS}

The following conventions will be used throughout this formulation.

(1) Mechanical parts will be represented by a number of joints and links. The goal is to relate geometric dimensions by imposing constraints inherent in the mathematics of kinematic pairs. For example, consider the mechanical part shown in Fig. 1a. By choosing the appropriate types of joints, the designer allows for the distortion of the part in a particular manner. For example, two sliding joints $\left(\mathrm{J}_{2}\right.$ and $\left.\mathbf{J}_{3}\right)$, a revolute joint $\mathbf{J}_{4}$, and spherical joints $\left(\mathbf{J}_{1}, \mathbf{J}_{5}, \mathbf{J}_{6}, \mathbf{J}_{7}\right.$, and $\left.\mathbf{J}_{8}\right)$ are introduced as shown in Fig. 1b. The kinematic model of the part is shown in Fig. 1c where the proposed links are numbered 0 through 6 and the mechanical part has been modeled as a mechanism.

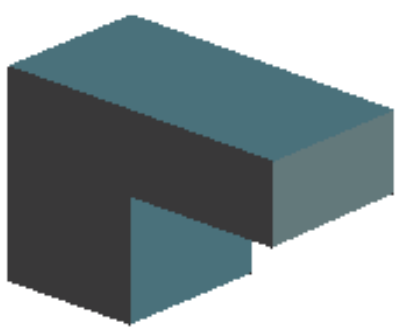

Fig. 1 (a) Mechanical part

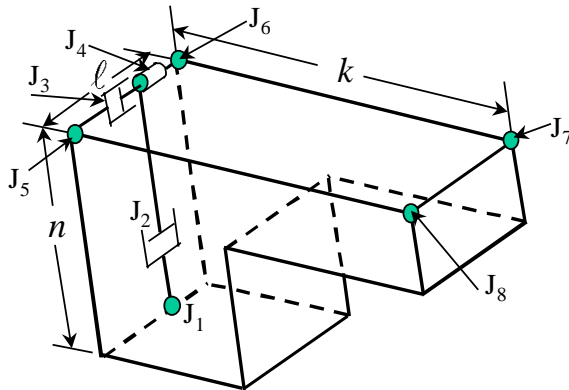

(b) Modeling the part as joints and links (c) The mechanism

representation of the part

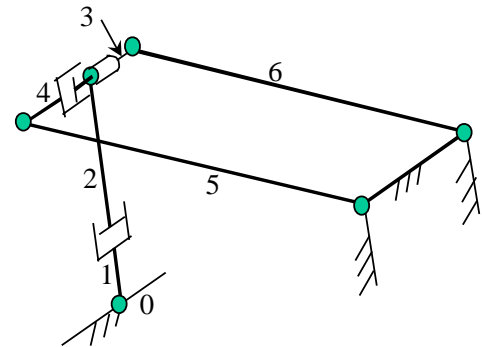


(2) A mechanism will be transformed into a graph. Each body is defined as a node and a kinematic joint is defined as an edge. For example, the scissors jack shown in Fig. 2a will be transformed into a graph representation as shown in Fig. $2 b$.

(3) A Graph representation will be cut to produce a tree structure. For example, the graph of Fig. $2 \mathrm{~b}$ is cut as shown in Fig. $2 \mathrm{c}$ to obtain a tree.
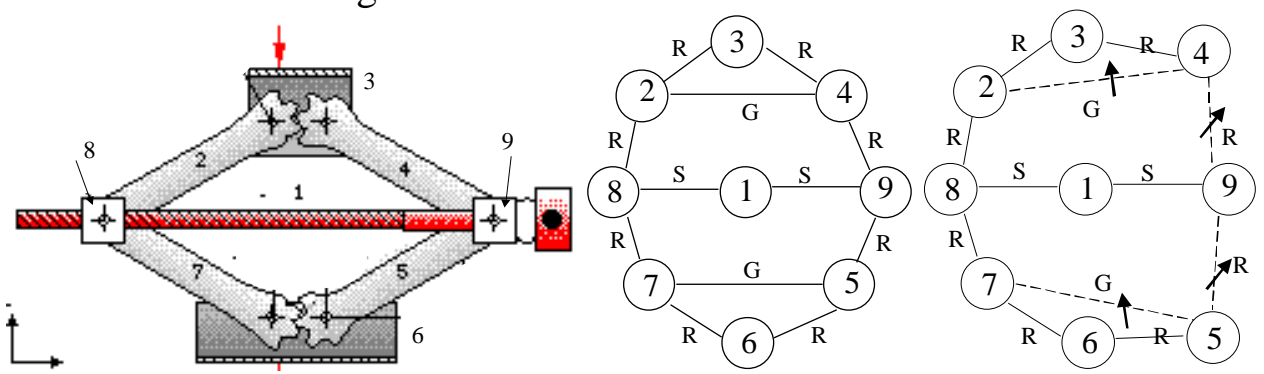

Fig. 2 (a) Scissors jack (b) A graph representation (c) Cutting joints to form a tree

(3) Vector notation used throughout this formulation is introduced in this section. In order to facilitate the forthcoming discussion, Fig. 3 illustrates the nomenclature used throughout.

Define the vectors $\mathbf{a}_{i}$ and $\mathbf{a}_{j}$ as nonzero vectors fixed in the bodies $i$ and $j$, respectively. Define the vectors $\mathbf{r}_{i}$ and $\mathbf{r}_{j}$ as the global position vectors extending from the origin of the global reference frame to the origin of the body reference frame.

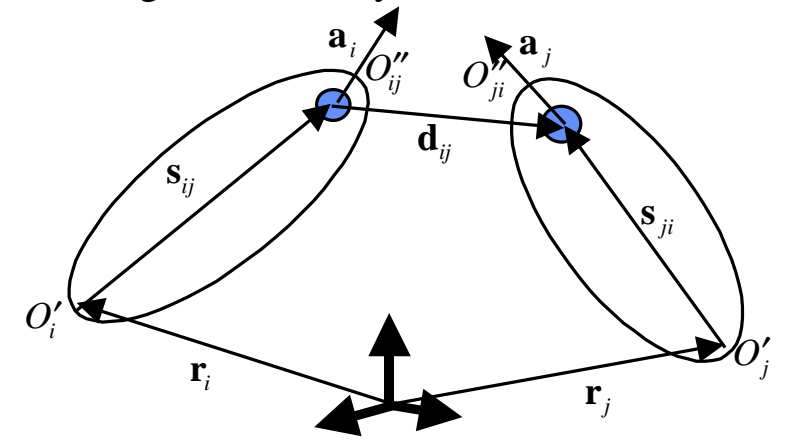

Fig. 3 Identification of coordinate systems

Define the vectors $\mathbf{s}_{i j}$ and $\mathbf{s}_{j i}$ as the joint-attachment vectors in the body reference frame, where these vectors will be maintained as variables such that their variations will be automatically computed.

\section{PROBLEM FORMULATION}

In this section, the results obtained by Zou et al. (1997) are first briefly reviewed. The expansion of the formulation is then introduced in terms of the representation of 3D parts and mechanisms. Throughout this derivation, the position vector embedded in each link and its orientation matrix are maintained as variables. The goal is to obtain linearized equation characterizing the assembly (i.e., mechanical part)

\subsection{Review of Constraints (Zou, et al. 1996)}

A spherical joint is a type of constraint that requires a pair of points on two bodies to coincide. A necessary and sufficient condition for $O_{i j}^{\prime \prime}$ and $O_{j i}^{\prime \prime}$, to coincide is that $\mathbf{d}_{i j}=\mathbf{0}$; i.e.,

$$
\boldsymbol{\Phi}^{s}\left(O_{i j}^{\prime \prime}, O_{j i}^{\prime \prime}\right)=\mathbf{r}_{j}+\mathbf{s}_{j i}-\mathbf{r}_{i}-\mathbf{s}_{i j}=\mathbf{0}
$$


The vectors $\mathbf{s}_{i j}$ and $\mathbf{s}_{j i}$ can be written as $\mathbf{s}_{i j}=\mathbf{A} i \mathbf{s}_{i j}^{\prime}$ and $\mathbf{s}_{j i}=\mathbf{A}_{j} \mathbf{s}_{j i}^{\prime}$. The variation of the joint position vectors can be written as

$$
\delta \mathbf{s}_{i j}=-\widetilde{\mathbf{s}}_{i j} \delta \boldsymbol{\pi}_{i}+\mathbf{A}_{i} \delta \mathbf{s}_{i j}^{\prime}
$$

Taking the variation of Eq. (1) and substituting for $\delta \mathbf{s}_{i j}$ and $\delta \mathbf{s}_{j i}$ yields

$$
\delta \Phi^{s}=\delta \mathbf{r}_{j}-\delta \mathbf{r}_{i}-\widetilde{\mathbf{s}}_{j i} \delta \boldsymbol{\pi}_{j}+\mathbf{A}_{j} \delta \mathbf{s}_{j i}^{\prime}+\widetilde{\mathbf{s}}_{i j} \delta \boldsymbol{\pi}_{i}-\mathbf{A}_{i} \delta \mathbf{s}_{j i}^{\prime}
$$

where the matrices $\mathbf{A}_{i}$ and $\mathbf{A}_{j}$ represent the direction cosine matrices from link reference frame $x^{\prime} y^{\prime} z^{\prime}$ to the global reference frame $x y z$; the symbol $(\sim)$ called tilde, is used to denote a skew-symmetric matrix generated by its corresponding vector (e.g., $\widetilde{\mathbf{a}}$ is the skew symmetric matrix generated from the vector $\mathbf{a}=\left[\begin{array}{lll}a_{x} & a_{y} & a_{z}\end{array}\right]^{T}$ ); the matrix $\pi$ can be generated from the elements of $\pi=\delta \mathbf{A} \mathbf{A}^{T}$.

A distance constraint requires a specified distance between a pair of points. A necessary and sufficient condition that the distance between $O_{i j}^{\prime \prime}$ and $O_{j i}^{\prime \prime}$, shown in Fig. 1, be equal to $\ell \neq 0$ is that) is formulated as

$$
\boldsymbol{\Phi}^{D}\left(O_{i j}^{\prime \prime}, O_{i j}^{\prime \prime}, \ell\right)=\mathbf{d}_{i j}^{T} \mathbf{d}_{i j}-\ell^{2}=\mathbf{0}
$$

and its variation is

$$
\delta \Phi^{D}=2 \mathbf{d}_{i j}^{T} \delta \mathbf{d}_{i j}=2 \mathbf{d}_{i j}^{T}\left[\delta \mathbf{r}_{j}-\delta \mathbf{r}_{i}-\widetilde{\mathbf{s}}_{j i} \delta \boldsymbol{\pi}_{j}+\mathbf{A}_{j} \delta \mathbf{s}_{j i}^{\prime}+\widetilde{\mathbf{s}}_{i j} \delta \boldsymbol{\pi}_{i}-\mathbf{A}_{i} \delta \mathbf{s}_{i j}^{\prime}\right]
$$

where the variation of $\mathbf{d}_{i j}$ is

$$
\delta \mathbf{d}_{i j}=\delta \mathbf{r}_{j}-\delta \mathbf{r}_{i}-\widetilde{\mathbf{s}}_{j i} \delta \boldsymbol{\pi}_{j}+\mathbf{A}_{j} \delta \mathbf{s}_{j i}^{\prime}+\widetilde{\mathbf{s}}_{i j} \delta \boldsymbol{\pi}_{i}-\mathbf{A}_{i} \delta \mathbf{s}_{i j}^{\prime}
$$

Combination of joints is performed in the analysis to simplify computations. Consider, for example, the translational and distance constraints: If the two joints are connected to each other in one branch, then they can be combined in the analysis and will be denoted by $T-D$. The constraint equation that characterizes this joint is

$$
\boldsymbol{\Phi}^{T-D}=\mathbf{I}_{i j}^{T} \mathbf{I}_{i j}-\ell_{T-D}^{2}
$$

where

$$
\mathbf{I}_{i j}=\mathbf{d}_{i j}-\mathbf{h}_{i j} q_{3}
$$

where

$$
\mathbf{h}_{i j}=\mathbf{A}_{i} \mathbf{C}_{i j}\left[\begin{array}{lll}
0 & 0 & 1
\end{array}\right]^{T}
$$

Substituting Eq. (9) into (8) and subsequently into (7) and using the identities derived, the variation of $\boldsymbol{\Phi}^{T-D}$ is

$$
\delta \boldsymbol{\Phi}^{T-D}=2 \mathbf{I}_{i j}^{T}\left[\delta \mathbf{r}_{j}+\mathbf{A}_{j} \delta \mathbf{s}_{j i}^{\prime}-\widetilde{\mathbf{s}}_{j i} \delta \boldsymbol{\pi}_{j}-\delta \mathbf{r}_{i}-\mathbf{A}_{i} \delta \mathbf{s}^{\prime}+\left(\widetilde{\mathbf{s}}_{i j}+\widetilde{\mathbf{h}}_{i j} q_{3}\right) \delta \boldsymbol{\pi}_{i}+\widetilde{\mathbf{h}}_{i j} q_{3} \delta \boldsymbol{\xi}_{i j}-\mathbf{h}_{i j} \delta q_{3}\right]-2 \ell_{T-D} \delta \ell_{T-D}
$$

\subsection{Jacobian Matrices}

The Jacobian matrix of a composite $T$ - $D$ joint in Cartesian space can be written as

$$
\boldsymbol{\Phi}_{\hat{\mathbf{z}}_{j}}^{T-D}=\left[\begin{array}{ll}
\boldsymbol{\Phi}_{\mathbf{r}_{j}} & \boldsymbol{\Phi}_{\boldsymbol{\pi}_{j}}
\end{array}\right]\left[\begin{array}{cc}
\mathbf{I} & -\widetilde{\mathbf{r}}_{j} \\
\mathbf{0} & \mathbf{I}
\end{array}\right]
$$

where the symbol $\Phi_{\hat{\mathbf{z}}_{j}}$ denotes the Jacobian matrix of the constraint function $\boldsymbol{\Phi}$ with respect to the coordinate space of $\hat{\mathbf{z}}$ (i.e., $\left[\partial \Phi_{i} / \partial \hat{\mathbf{z}}_{j}\right]$ ), and can be written as

The Jacobian matrix is

$$
\boldsymbol{\Phi}_{\mathbf{z}_{j}}^{T-D}=\left[\begin{array}{ll}
2 \mathbf{I}_{i j}^{T} & -2 \mathbf{I}_{i j}^{T}\left(\widetilde{\mathbf{s}}_{j i}+\widetilde{\mathbf{r}}_{j}\right)
\end{array}\right]
$$




$$
\boldsymbol{\Phi}_{q_{3}}^{T-D}=-2 \mathbf{I}_{i j}^{T} \mathbf{h}_{i j}
$$

\subsection{Velocity Transformation Matrices of Combined Joints}

A Spherical-Translational (denoted by $S-T$ ) joint has four relative degrees of freedom

$\mathbf{q}_{S-T}=\left[\begin{array}{ll}\mathbf{q}_{1} & q_{2}\end{array}\right]^{T}$, three for the spherical joint and one for the translational joint. Three relative rotational coordinates of the spherical joint can be expressed using Euler parameters to avoid orientation-associated singularities. The Euler parameters are denoted by $e_{0}, e_{1}, e_{2}$, and $e_{3}$ such that the Euler parameter vector is defined by

$$
\mathbf{p}_{1}=\left[\begin{array}{llll}
\mathrm{e}_{0} & \mathrm{e}_{1} & \mathrm{e}_{2} & \mathrm{e}_{3}
\end{array}\right]^{T}
$$

Since a translational joint does not have a rotational degree of freedom, the transformation matrix $\mathbf{A}_{i j}^{\prime \prime}$ of the $S-T$ joint is simply the same as that of a spherical joint, thus

$$
\mathbf{A}_{i j}^{\prime \prime}=\mathbf{A}_{s p h}^{\prime \prime}=\mathbf{E}_{j} \mathbf{G}_{j}^{T}
$$

where $\mathbf{E}_{j}$ and $\mathbf{G}_{j}$ are the Euler parameters semi-rotation matrices defined by Haug (1989) as

and

$$
\begin{gathered}
\mathbf{E}_{j}=\left[-\mathbf{e}_{j}, \tilde{\mathbf{e}}_{j}+\mathrm{e}_{j 0} \mathbf{I}\right]=\left[\begin{array}{cccc}
-\mathrm{e}_{1} & \mathrm{e}_{0} & -\mathrm{e}_{3} & \mathrm{e}_{2} \\
-\mathrm{e}_{2} & \mathrm{e}_{3} & \mathrm{e}_{0} & -\mathrm{e}_{1} \\
-\mathrm{e}_{3} & -\mathrm{e}_{2} & \mathrm{e}_{1} & \mathrm{e}_{0}
\end{array}\right] \\
\mathbf{G}_{j}=\left[-\mathbf{e}_{j},-\tilde{\mathbf{e}}_{j}+\mathrm{e}_{j 0} \mathbf{I}\right]=\left[\begin{array}{cccc}
-\mathrm{e}_{1} & \mathrm{e}_{0} & \mathrm{e}_{3} & -\mathrm{e}_{2} \\
-\mathrm{e}_{2} & -\mathrm{e}_{3} & \mathrm{e}_{0} & \mathrm{e}_{1} \\
-\mathrm{e}_{3} & \mathrm{e}_{2} & -\mathrm{e}_{1} & \mathrm{e}_{0}
\end{array}\right]
\end{gathered}
$$

and the transformation matrix $\mathbf{C}_{i j}$ can be represented as

$$
\mathbf{C}_{i j}=\mathbf{E}_{j} \mathbf{G}_{j}^{T}
$$

The velocity transformation matrix $\mathbf{B}_{j}$ can be expressed as

$$
\mathbf{B}_{j}^{S-T}=\left[\begin{array}{ll}
\mathbf{B}_{s p h} & \mathbf{B}_{\text {tran }}
\end{array}\right]_{6 \times 5}
$$

where $\mathbf{B}_{s p h}$ is the velocity transformation for the spherical joint

$$
\mathbf{B}_{s p h}=\left[\begin{array}{c}
\widetilde{\mathbf{r}}_{j} \\
\mathbf{I}
\end{array}\right] \mathbf{A}_{i} \mathbf{C}_{i j} 2 \mathbf{E}_{j}=\left[\begin{array}{c}
\widetilde{\mathbf{r}}_{j} \\
\mathbf{I}
\end{array}\right] \mathbf{A}_{1} \mathbf{C}_{12 S-T} 2 \mathbf{E}_{j 6 \times 4}
$$

and $\mathbf{B}_{\text {tran }}$ is the velocity transformation for the translational joint

$$
\mathbf{B}_{\text {tran }}=\left[\begin{array}{ll}
\mathbf{g}_{i j} & \mathbf{0}
\end{array}\right]^{T}
$$

Velocity transformation matrices are used to represent the variation of one body with respect to another. Assume bodies $i$ and $j$ are connected by two joints; after joint 1 is cut, body $i$ is the inboard body of body $j$. In order to obtain a representation of one vector in terms of another in state vector form, and since the variation $\delta \mathbf{r}_{j}=\delta \mathbf{r}_{i}+\delta \mathbf{s}_{i j}+\delta \mathbf{d}_{i j}$, then substitute for $\delta \mathbf{d}_{i j}$ and $\delta \mathbf{s}_{i j}$ and use the relationship $\tilde{\mathbf{a}} \mathbf{b}=-\tilde{\mathbf{b}} \mathbf{a}$ yields

$$
\delta \mathbf{r}_{j}=\delta \mathbf{r}_{i}+\delta \tilde{\pi}_{i} \mathbf{s}_{i j}+\mathbf{A}_{i} \delta \mathbf{s}_{i j}^{\prime}+\delta \tilde{\pi}_{i} \mathbf{d}_{i j}-\widetilde{\mathbf{d}}_{i j} \delta \xi_{i j}^{\prime}+\frac{\delta \mathbf{d}_{i j}}{\delta \mathbf{q}_{j}} \delta \mathbf{q}_{j}
$$

Collecting terms and simplifying yields 


$$
\delta \mathbf{r}_{j}=\delta \mathbf{r}_{i}+\delta \tilde{\boldsymbol{\pi}}_{i}\left(\mathbf{r}_{j}-\mathbf{r}_{i}\right)+\mathbf{A}_{i} \delta \mathbf{s}_{i j}^{\prime}-\widetilde{\mathbf{d}}_{i j} \delta \xi_{i j}^{\prime}+\frac{\delta \mathbf{d}_{i j}}{\delta \mathbf{q}_{j}} \delta \mathbf{q}_{j}
$$

Adding a common term $\left(\widetilde{\mathbf{r}}_{j} \delta \boldsymbol{\pi}_{j}\right)$ to both sides of Eq. (23), and using the above identities yields

$$
\delta \mathbf{r}_{j}+\widetilde{\mathbf{r}}_{j} \delta \boldsymbol{\pi}_{j}=\delta \mathbf{r}_{i}+\widetilde{\mathbf{r}}_{i} \delta \boldsymbol{\pi}_{i}+\mathbf{A}_{i} \delta \mathbf{s}_{i j}^{\prime}-\widetilde{\mathbf{d}}_{i j} \delta \xi_{i j}^{\prime}+\left(\frac{\delta \mathbf{d}_{i j}}{\delta \mathbf{q}_{j}}+\widetilde{\mathbf{r}}_{j} \mathbf{H}_{j}\right) \delta \mathbf{q}_{j}+\widetilde{\mathbf{r}}_{j} \delta \xi_{i j}^{\prime}
$$

where $\mathbf{H}_{j}\left(\mathbf{A}_{i}, \mathbf{q}_{j}\right)$ is a transformation matrix that depends on the orientation of body $i$ and on the relative coordinates $\delta \mathbf{q}_{j}$, which is defined for each type of joint such that $\delta \boldsymbol{\pi}_{i j}=\mathbf{H}_{j}\left(\mathbf{A}_{i}, \mathbf{q}_{j}\right) \delta \mathbf{q}_{j}$. The virtual rotation is expressed by $\delta \boldsymbol{\pi}_{j}=\delta \boldsymbol{\pi}_{i}+\delta \xi_{i j}+\mathbf{H}_{j} \delta \mathbf{q}_{j}$. Equation (24) and the virtual rotation are combined in matrix form as

$$
\left[\begin{array}{c}
\delta \mathbf{r}_{j}+\widetilde{\mathbf{r}}_{j} \delta \boldsymbol{\pi}_{j} \\
\delta \boldsymbol{\pi}_{j}
\end{array}\right]=\left[\begin{array}{c}
\delta \mathbf{r}_{i}+\widetilde{\mathbf{r}}_{i} \delta \boldsymbol{\pi}_{i} \\
\delta \boldsymbol{\pi}_{i}
\end{array}\right]+\left[\begin{array}{c}
\mathbf{A}_{i} \\
\mathbf{0}
\end{array}\right] \delta \mathbf{s}_{i j}^{\prime}+\left[\begin{array}{c}
\left(\partial \mathbf{d}_{i j} / \partial \mathbf{q}_{j}\right)+\widetilde{\mathbf{r}}_{i} \mathbf{H}_{j} \\
\mathbf{H}_{j}
\end{array}\right] \delta \mathbf{q}_{j}+\left[\begin{array}{c}
\widetilde{\mathbf{r}}_{j}-\tilde{\mathbf{d}}_{i j} \\
\mathbf{I}
\end{array}\right] \delta \xi_{i j}
$$

In state-vector notation, Eq. (25) is written as

$$
\delta \tilde{\mathbf{z}}_{j}=\delta \tilde{\mathbf{z}}_{i}+\mathbf{B}_{j} \delta \mathbf{q}_{j}+\mathbf{M}_{i} \delta \mathbf{s}_{i j}^{\prime}+\mathbf{N}_{j} \delta \xi_{i j}^{\prime}
$$

where

$$
\mathbf{M}_{i}=\left[\begin{array}{ll}
\mathbf{A}_{i} & \mathbf{0}
\end{array}\right]^{T} \quad \text { and } \quad \mathbf{N}_{j}=\left[\begin{array}{ll}
\widetilde{\mathbf{r}}_{j}-\widetilde{\mathbf{d}}_{i j} & \mathbf{I}
\end{array}\right]^{T}
$$

This state representation characterized by Eq. (26) will be used in obtaining a linear set of equations that can automatically be solved for an appropriate configuration.

\section{COMPUTING A SOLUTION FROM A NON-SQUARE JACOBIAN}

The general case of the variation of the constraint $\Phi$ in linearized form can be written as

$$
\delta \Phi=\Phi_{\hat{\mathbf{z}}_{j}} \mathbf{B}_{j} \delta \mathbf{q}_{j}+\Phi_{\hat{\mathbf{z}}_{j}} \mathbf{M}_{i} \delta \mathbf{s}_{i j}^{\prime}+\Phi_{\widehat{\mathbf{z}}_{j}} \mathbf{N}_{j} \delta \xi_{i j}+\Phi_{\mathbf{s}_{i j}} \delta \mathbf{s}_{i j}+\Phi_{\xi_{i j}} \delta \xi_{i j}
$$

or simply represented by

$$
\Phi_{\mathbf{q}} \delta \mathbf{q}=-\Phi
$$

When a given initial estimate does not satisfy the constraint equations $\boldsymbol{\Phi}$, the linearized variational constraints (Eq. 27) are simultaneously solved to obtain an admissible solution. Note that the number of variables is more than the number of constraints. Since the Jacobian of $\Phi(\mathbf{q})$ is not square, the problem of obtaining an assembled configuration can be solved using the Moore-Penrose pseudo inverse (Abdel-Malek and Yeh 1997). Starting with an initial guess $\mathbf{q}^{1}$, the new generalized coordinates are calculated by evaluating

$$
\Delta \mathbf{q}=\Phi_{\mathbf{q}}^{*}(-\Phi)
$$

where $\Phi_{\mathbf{q}}^{*}$ is the Moore-Penrose pseudo inverse of the Jacobian $\Phi_{\mathbf{q}}=\left[\partial \Phi_{i} / \partial q_{j}\right]$, defined by

$$
\Phi_{\mathbf{q}}^{*}=\Phi_{\mathbf{q}}^{T}\left(\Phi_{\mathbf{q}} \Phi_{\mathbf{q}}^{T}\right)^{-1}
$$

The new set of generalized coordinates is iteratively computed as $\mathbf{q}^{(i+1)}=\mathbf{q}^{(i)}+\Delta \mathbf{q}^{(i)}$ until the constraint function $\|\Phi\|<\varepsilon$ is satisfied. The method converges to a solution $\mathbf{q}^{*}$ within a few iterations because of its quadratic rate of convergence. This method will yield a solution set $\mathbf{q}$ that is closest to the original configuration but with changed dimensions. The final solution characterizes an assembled mechanism that satisfies all kinematic relations imposed by the designer. This new mechanism can now be converted back to a mechanical part. The overall method is illustrated in Fig. 4. 


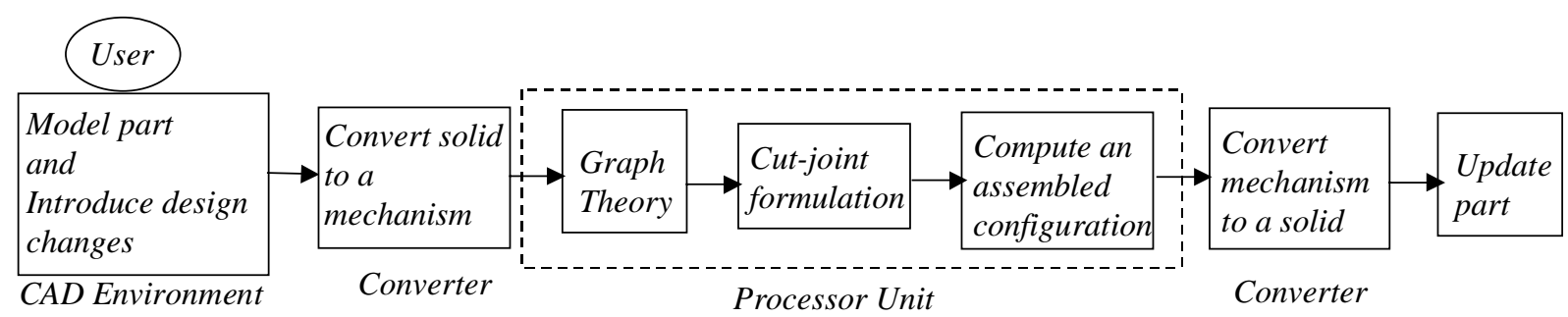

\section{EXAMPLE I}

Fig. 4 Block diagram of the overall methodology

Consider the mechanical part shown in Fig. 5a and its cross section shown in Fig. 5b. Due to mating part requirements, an increase in the slot radius $r$ to $r+d r$ is introduced. It required, however, to maintain the distance $d_{1}$ as constant due to assembly requirements but changes in the dimension $d_{2}$ and the angle $\beta$ of the slot are allowed. Although this is a 3D part, the resulting problem is planar as the mechanism is planar.
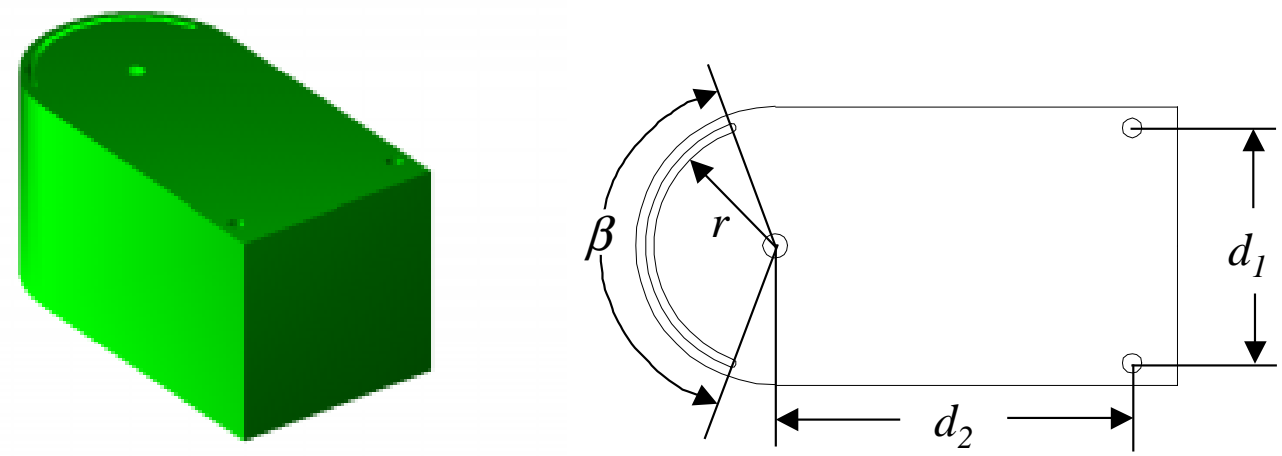

Fig. 5 (a) A mechanical part and its cross section (b) A cross section of the part

To kinematically model this mechanical part, five revolute joints $J_{1}, J_{2}, J_{3}, J_{5}$, and $J_{6}$, and two prismatic joints $J_{4}$ and $J_{7}$ are introduced as shown in Fig. 6. The prismatic joint mandates a horizontal movement (change) of the dimension $d_{2}$ while maintaining the dimension $d_{1}$. The revolute joint at $J_{1}$ is fixed to the ground (i.e., does not translate). Links 0,1,2,3,4, and 5 are introduced and the connectivity is shown where Link 0 is ground. The graph representation of the kinematic model is shown in Fig. 7a.

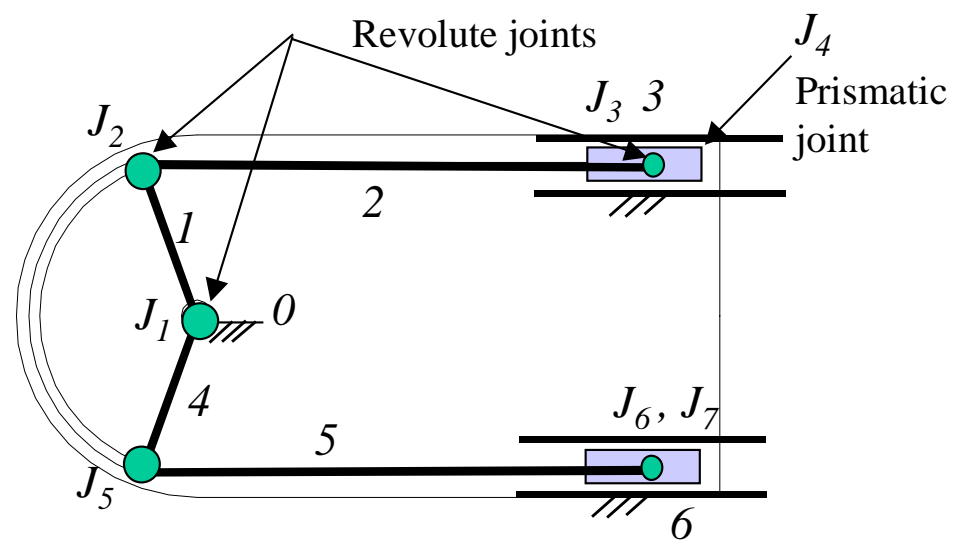


Fig. 6. Kinematic modeling of the mechanical part
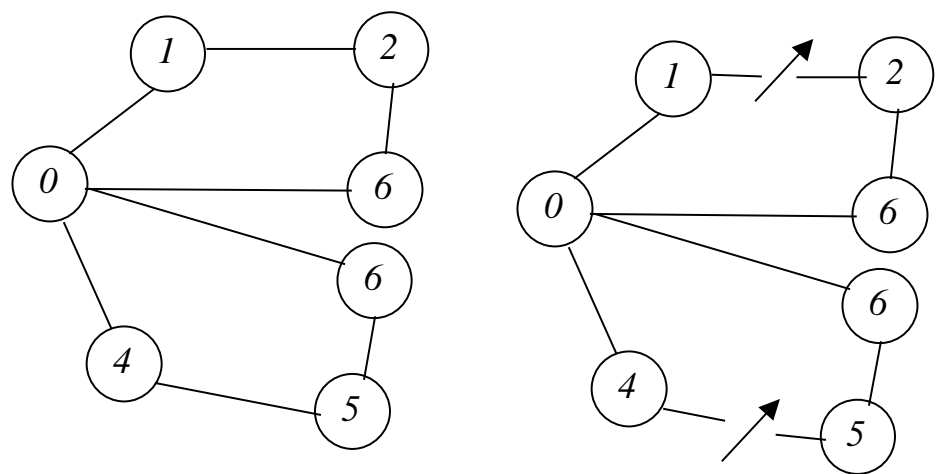

Fig. 7. (a) Graph representation of the mechanical part (b) after cutting

Due to the design change, a variation is to occur in the vector of Euler parameters $\mathbf{p}_{01}$ associated with the transformation matrix $\mathbf{C}_{01}$. To form a spanning tree, the joint connecting links 1 and 2 and that connecting link 4 and 5 are cut, where the bodies do not remain connected after cutting the joint as shown in Fig. 7b. Note that both sides are symmetric. Since a joint was cut, a constraint function is introduced such that

$$
\boldsymbol{\Phi}^{r}=\mathbf{r}_{2}+\mathbf{S}_{21}-\mathbf{s}_{12}-\mathbf{r}_{1}=\mathbf{0}
$$

where $\mathbf{s}_{12}$ is the joint-position vector in the reference frame of link 1; and $\mathbf{s}_{21}$ is the jointposition vector in the reference frame of link 2. The Jacobian matrix of the cut-joint in the reference frame of link 1, written in Cartesian coordinate space is

$$
\boldsymbol{\Phi}_{\mathbf{\mathbf { z }}_{1}}^{r}=\left[\begin{array}{ll}
-\mathbf{I} & \widetilde{\mathbf{r}}_{1}+\widetilde{\mathbf{s}}_{12}
\end{array}\right]
$$

In order to transform the Jacobian matrix from Cartesian coordinate space to joint coordinate space, the velocity transformation matrices are used such that

$$
\boldsymbol{\Phi}_{\mathbf{q}}^{r}=\left[\begin{array}{lll}
\boldsymbol{\Phi}_{\mathbf{z}_{1}}^{r} \mathbf{B}_{\text {rev }} & \boldsymbol{\Phi}_{\mathbf{z}_{2}}^{r} \mathbf{B}_{\text {slid }} & \boldsymbol{\Phi}_{\mathbf{z}_{2}}^{r} \mathbf{B}_{\text {rev }}
\end{array}\right]
$$

where the velocity transformation matrix of the revolute joint is $\mathbf{B}_{\text {rev }}=\left[\begin{array}{ll}\widetilde{\mathbf{r}}_{1} \mathbf{h}_{01} & \mathbf{h}_{01}\end{array}\right]^{T}$, and $\mathbf{h}_{01}=\mathbf{A}_{0} \mathbf{C}_{01}\left[\begin{array}{lll}0 & 0 & 1\end{array}\right]^{T}$ is the unit vector along the rotational joint axis $\mathbf{z}_{01}^{\prime \prime}$ in the global frame. Using the linearized equations of Section 4, the iterative algorithm is employed until constraint violations are satisfied, where $\mathbf{p}_{03}$ is the vector of Euler parameters associated with $\mathbf{C}_{03}$ (transformation matrix from translational joint coordinates to ground reference frame); $\mathbf{p}_{32}$ is the vector of Euler parameters associated with $\mathbf{C}_{32}$. The new computed configuration of the mechanism is shown in Fig. 8a. The original part is shown in Fig. $8 \mathrm{~b}$ and the updated geometry in Fig. 8c. 


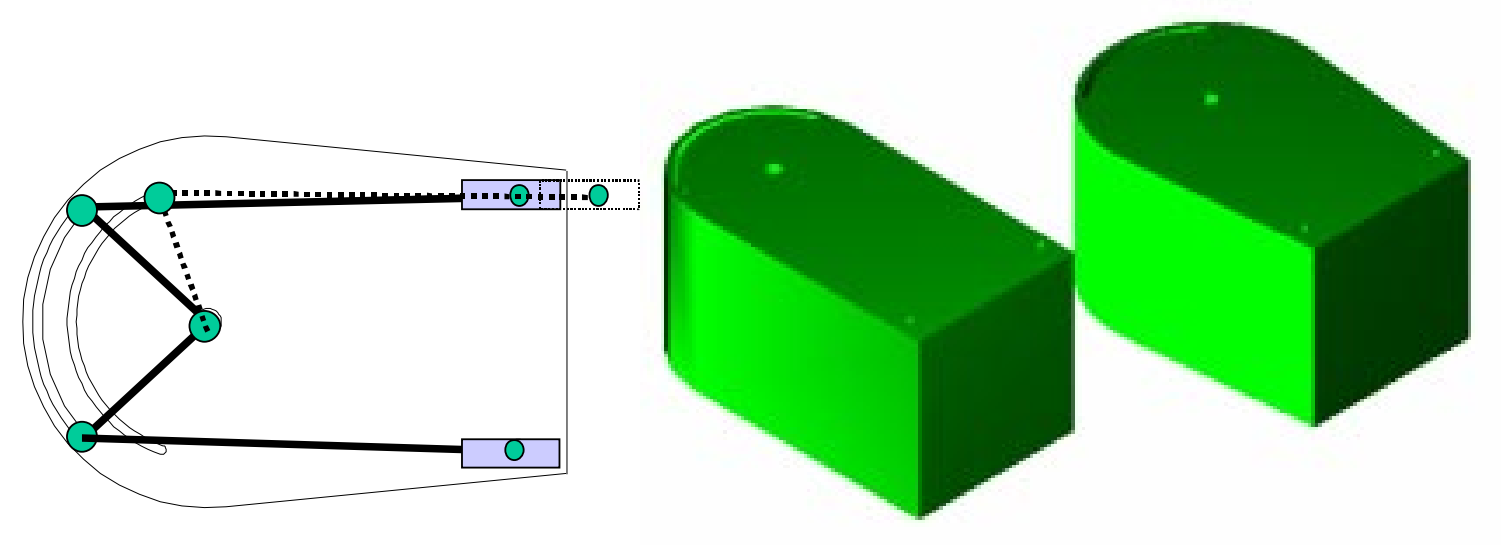

Fig. 8. (a) Kinematic change in the mechanism (b) the original part (c) the new computed part

\section{EXAMPLE II}

Consider the mechanical part shown in Fig. 9a. In order to compensate for a change in the dimensions of a mating part (not shown), the dimension $n$ is required to change to $n+\partial n$. However, a constraint is introduced such that to keep the distance $d$ in Fig. 9a unchanged.

The designer selects the type of joints and their locations. Joints $J_{2}, J_{4}$, and $J_{6}$ are translational joints, $J_{3}$ and $J_{5}$ are distance joints, and $J_{1}$ is a spherical joint. The distance joint followed by a translational joint, in this case, specifies a minimum distance that must be maintained but also allows for deformation in the part allowing the distance to be kept. The system is carefully chosen to allow for distortions in certain geometric parameters. The model comprises two composite Translational-Distance joints $(T-D)$ and one composite SphericalTranslational $(S-T)$ joint as shown in Fig. 9 b.

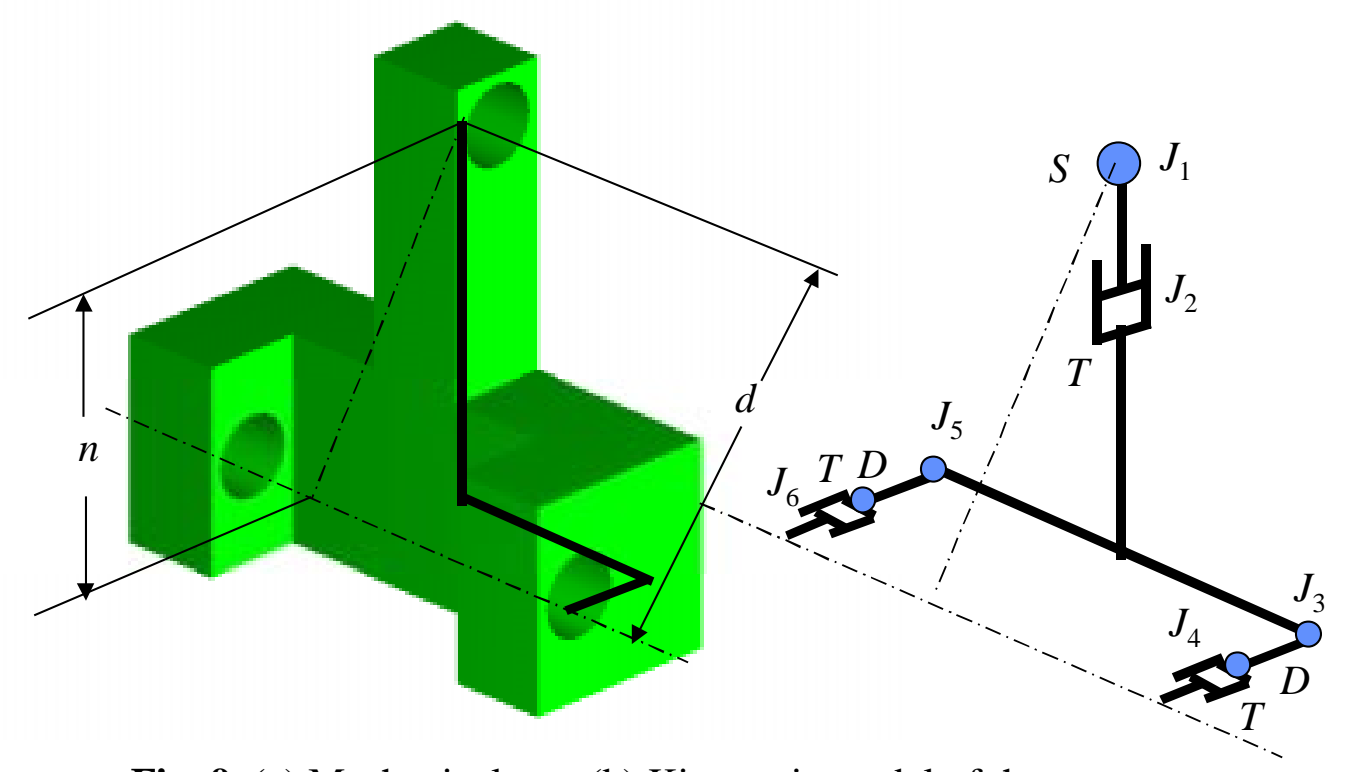

Fig. 9 (a) Mechanical part (b) Kinematic model of the part 
To form a spanning tree structure, the two composite $T-D$ joints are cut. The graph representation of the system before cutting joints is shown in Fig. 10a and the tree after cutting is shown in Fig. 10b.

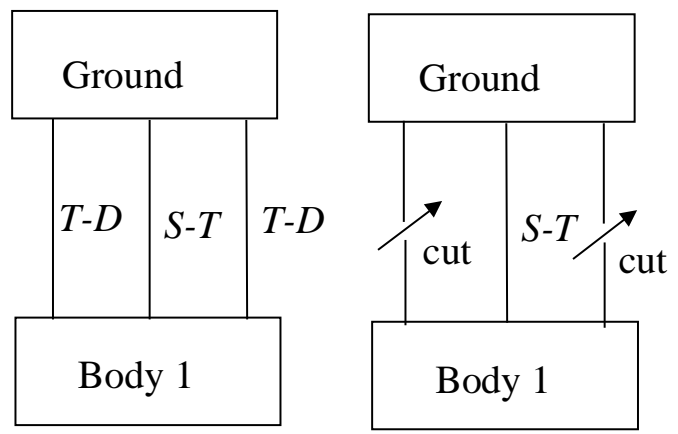

Fig. 10 (a) Graph representation of the kinematic model (b) Cutting joints

Since the $S$ - $T$ joint connects the two entities (ground and body) with a spherical joint and a translational joint, define the vector $\mathbf{s}_{{ }^{\prime 2 S-T}}^{\prime}$ as the $S$ - $T$ joint-attachment vector in ground reference frame, and let $\ell_{\mathrm{T}-\mathrm{D}}$ be the constraint distance.

Using the proposed formulation, the linear systems of equations in terms of the Jacobians for this mechanism are written as

$$
\left[\begin{array}{cc}
\boldsymbol{\Phi}_{\mathbf{q}_{S-T}}^{S-T} & \mathbf{0} \\
\boldsymbol{\Phi}_{\mathbf{q}_{T-D}^{T-D}}^{T-} & \boldsymbol{\Phi}_{q_{3}}^{T-D}
\end{array}\right]\left[\begin{array}{c}
\delta \mathbf{q}_{S-T} \\
\delta q_{3}
\end{array}\right]=\left[\begin{array}{c}
-\boldsymbol{\Phi}^{S-T} \\
-\boldsymbol{\Phi}^{T-D}
\end{array}\right]
$$

where $\boldsymbol{\Phi}_{\mathbf{q}_{S-T}^{S-T}}^{S}$ and $\boldsymbol{\Phi}_{\mathbf{q}_{S-T}^{T-D}}^{T-2}$ are the Jacobian matrices in joint coordinate space, and are transformed from the Cartesian space $\Phi_{\hat{\mathbf{z}}_{j}}$ by using the velocity transformation matrix $\mathbf{B}$ as

$$
\begin{aligned}
\boldsymbol{\Phi}_{\mathbf{q}_{S-T}}^{S-T} & =\boldsymbol{\Phi}_{\hat{\mathbf{z}}_{j}}^{S-T} \mathbf{B}_{j}^{S-T} \\
\boldsymbol{\Phi}_{\mathbf{q}_{S-T}}^{T-D} & =\boldsymbol{\Phi}_{\hat{\mathbf{z}}_{j}}^{T-D} \mathbf{B}_{j}^{T-D}
\end{aligned}
$$

Estimates of transformation matrices $\mathbf{C}_{i j}$ (Joint coordinate to body reference frame) are

$$
\mathbf{C}_{12 S-T}=\left[\begin{array}{ccc}
0 & -1 & 0 \\
1 & 0 & 0 \\
0 & 0 & 1
\end{array}\right] \quad \mathbf{C}_{12 T-D}=\left[\begin{array}{ccc}
0 & 0 & 1 \\
1 & 0 & 0 \\
0 & 1 & 0
\end{array}\right] \quad \mathbf{C}_{21 T-D}=\left[\begin{array}{ccc}
0 & -1 & 0 \\
1 & 0 & 0 \\
0 & 0 & 1
\end{array}\right]
$$

and corresponding Euler parameters $\mathbf{P}_{i j}$ are computed as $\mathbf{P}_{12 \mathrm{~s}-\mathrm{T}}=\left[\begin{array}{lllll}0.707107 & 0 & 0 & 0.707107\end{array}\right]^{T}$ $\mathbf{P}_{\text {12T-D }}=\left[\begin{array}{llll}0.5 & 0.5 & 0.5 & 0.5\end{array}\right]^{T}$, and estimates of the generalized coordinates are $\mathbf{q}_{1}=\left[\begin{array}{llll}1 / \sqrt{2} & 0 & 0 & -1 / \sqrt{2}\end{array}\right]^{T}, q_{2}=-0.425$, and $q_{3}=-0.305$.

(1) The equation for allowing joint variables to automatically be computed must be determined. For a given design variation, the generalized coordinates $\mathbf{q}$ are allowed to vary and the linear system of equations is

$$
\boldsymbol{\Phi}_{\mathbf{z}_{2}} \mathbf{B}_{2}^{S-T} \delta \mathbf{q}_{S-T}+\Phi_{q_{3}} \delta q_{3}=-\Phi
$$

Equation (39) is solved for the joint variables using the iterative algorithm. This method yields a new configuration of the mechanism with only varying the generalized coordinates. This is the most direct method and yields results that are easily perceived by the designer prior to modeling. The part is updated accordingly. Results of performing this method are 
entered into column 3 of Table 1. Note that the initial assembly of the mechanism is entered in column 2 of Table 1. Also note that shaded regions indicate invariant constants while bold font indicates changed design parameters

(2) For a given design variation, only joint-attachment vectors $\mathbf{s}_{i j}^{\prime}$ are allowed to change. The resulting linear equation is

$$
\boldsymbol{\Phi}_{\hat{\mathbf{z}}_{2}} \mathbf{M}_{1} \delta \mathbf{s}_{12 S-T}^{\prime}+\boldsymbol{\Phi}_{\mathbf{s}_{12 T-D}^{\prime}} \delta \mathbf{s}_{12 T-D}^{\prime}+\boldsymbol{\Phi}_{\mathbf{s}_{21 T-D}^{\prime}} \delta \mathbf{s}_{21 T-D}^{\prime}=-\boldsymbol{\Phi}
$$

Location of the new coordinates of each joint is changed so as to redesign the mechanism.

(3) For a given design variation, joint-attachment vectors and the length $l_{T-D}$ are allowed to change. In this case, the variational equation is

$$
\boldsymbol{\Phi}_{\mathbf{z}_{2}} \mathbf{M}_{1} \delta \mathbf{s}_{12 S-T}^{\prime}+\boldsymbol{\Phi}_{\mathbf{s}_{12 T-D}^{\prime}} \delta \mathbf{s}_{12 T-D}^{\prime}+\boldsymbol{\Phi}_{\mathbf{s}_{21 T-D}^{\prime}} \delta \mathbf{s}_{21 T-D}^{\prime}+\boldsymbol{\Phi}_{l} \delta l=-\boldsymbol{\Phi}
$$

(4) If a complete redesign is sought, and the joint attachment vectors $\mathbf{S}_{i j}^{\prime}$ and generalized coordinates $\mathbf{q}$ are allowed to change, the equation to be solved is

$$
\boldsymbol{\Phi}_{\hat{\mathbf{z}}_{2}} \mathbf{B}_{2}^{S-T} \delta \mathbf{q}_{S-T}+\Phi_{q_{3}} \delta q_{3}+\Phi_{\hat{\mathbf{z}}_{2}} \mathbf{M}_{1} \delta \mathbf{s}_{12 S-T}^{\prime}+\Phi_{\mathbf{s}_{12 T-D}^{\prime}} \delta \mathbf{s}_{12 T-D}^{\prime}+\Phi_{\mathbf{s}_{21 T-D}^{\prime}} \delta \mathbf{s}_{21 T-D}^{\prime}=-\boldsymbol{\Phi}
$$

Keeping the joint attachment vectors $\mathbf{s}_{i j}$ and transformation matrices $\mathbf{C}_{i j}$ constant, the NewtonRaphson iteration method is used to update the generalized coordinates $\mathbf{q}$. The algorithm converges within 2 iterations and the set of new generalized coordinates are computed and entered into column 3 of Table 1 . Upon obtaining an assembled configuration (an admissible solution that satisfies the constraints), a second converter must be used to convert the mechanism to an updated mechanical part. For example, an admissible solution is shown in

\begin{tabular}{|c|c|c|c|c|c|}
\hline $\begin{array}{c}1 \\
\text { Variables }\end{array}$ & $\begin{array}{c}2 \\
\text { initial assembly }\end{array}$ & $\begin{array}{l}3 \\
\mathbf{q}\end{array}$ & $\begin{array}{c}4 \\
\mathbf{3} \\
\mathbf{s}_{i j}\end{array}$ & $\mathbf{s}_{i j} l_{R-S}^{5} l_{T-D}$ & $\begin{array}{c}6 \\
\mathbf{s}_{i j}, \mathbf{q}\end{array}$ \\
\hline $\mathbf{q}_{l}$ & {$\left[\begin{array}{c}0.6980 \\
-0.0320 \\
-0.0529 \\
-0.7137\end{array}\right]$} & {$\left[\begin{array}{c}0.6870 \\
0.0784 \\
-0.0912 \\
-0.7166\end{array}\right]$} & & & {$\left[\begin{array}{c}0.6995 \\
0.0199 \\
-0.0666 \\
-0.7112\end{array}\right]$} \\
\hline $\mathrm{q}_{2}$ & -0.4337 & -0.3638 & & & -0.4061 \\
\hline $\mathrm{q}_{3}$ & 0.2760 & 0.2349 & & & 0.2799 \\
\hline $\mathbf{S}_{I 2 S-T}$ & {$\left[\begin{array}{l}0.5011 \\
1.1162 \\
0.1998\end{array}\right]$} & {$\left[\begin{array}{c}0.5 \\
1.0 \\
0\end{array}\right]$} & {$\left[\begin{array}{c}0.5 \\
1.0 \\
0\end{array}\right]$} & {$\left[\begin{array}{c}0.5 \\
1.0 \\
0\end{array}\right]$} & {$\left[\begin{array}{c}0.5 \\
1.0 \\
0\end{array}\right]$} \\
\hline $\mathbf{S}_{2 I T-D}$ & {$\left[\begin{array}{c}0 \\
1.2675 \\
-0.3748\end{array}\right]$} & & {$\left[\begin{array}{c}0.0298 \\
1.2597 \\
-0.3931\end{array}\right]$} & {$\left[\begin{array}{c}0.0210 \\
1.2620 \\
-0.3876\end{array}\right]$} & {$\left[\begin{array}{c}0.0039 \\
1.2663 \\
-0.3772\end{array}\right]$} \\
\hline $\mathbf{S}_{2 I T-D}$ & {$\left[\begin{array}{c}0.07 \\
0.155 \\
-0.186\end{array}\right]$} & & {$\left[\begin{array}{c}0.0424 \\
0.1627 \\
-0.1645\end{array}\right]$} & {$\left[\begin{array}{c}0.0506 \\
0.1604 \\
-0.1709\end{array}\right]$} & {$\left[\begin{array}{c}0.0665 \\
0.1561 \\
-0.1831\end{array}\right]$} \\
\hline$l_{T-D}$ & 0.3742 & & & 0.3955 & \\
\hline $\mathbf{P}_{12 S-T}$ & {$\left[\begin{array}{c}0.7071 \\
0 \\
0 \\
0.7071\end{array}\right]$} & & & & \\
\hline
\end{tabular}
Fig. 11a, and the mechanical part in Fig. 11b. Other computed parts are shown in Fig. 12.

Table 1 Simulation results due to a change in $\mathbf{s}_{12 S-T}$ 


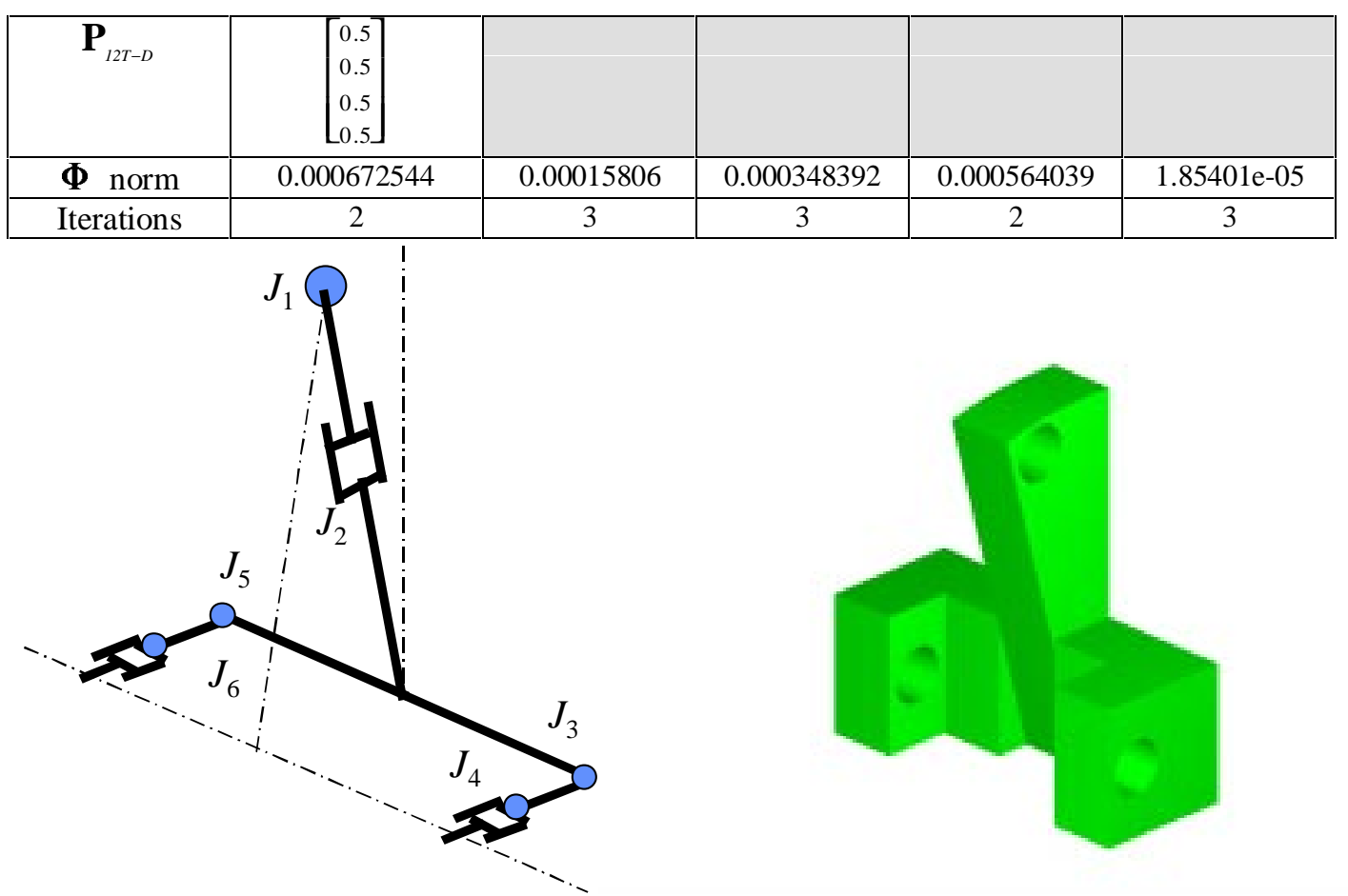

Fig. 11 (a) The mechanism after computing an assembled configuration (b) The equivalent redesigned part
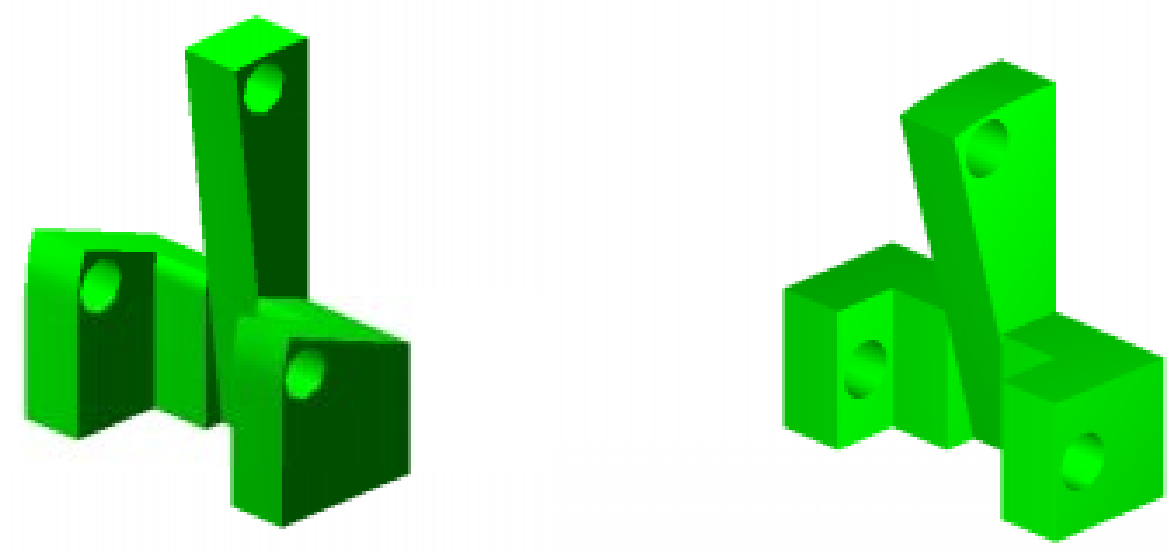

\section{EXAMPLE III}

Fig. 12 Other redesigned parts

To further illustrate, consider the mechanical part shown in Fig. 13a. The geometry of this part is represented by the mechanism shown in Fig. 13b, where one revolute joint, one spherical joint, one universal joint, and one prismatic joint have been introduced. The setup is such that the first and third links are subjected to torsion.

$$
\boldsymbol{\Phi}^{s p h}=\mathbf{r}_{2}+\mathbf{S}_{21}-\mathbf{S}_{12}-\mathbf{r}_{1}=\mathbf{0}
$$



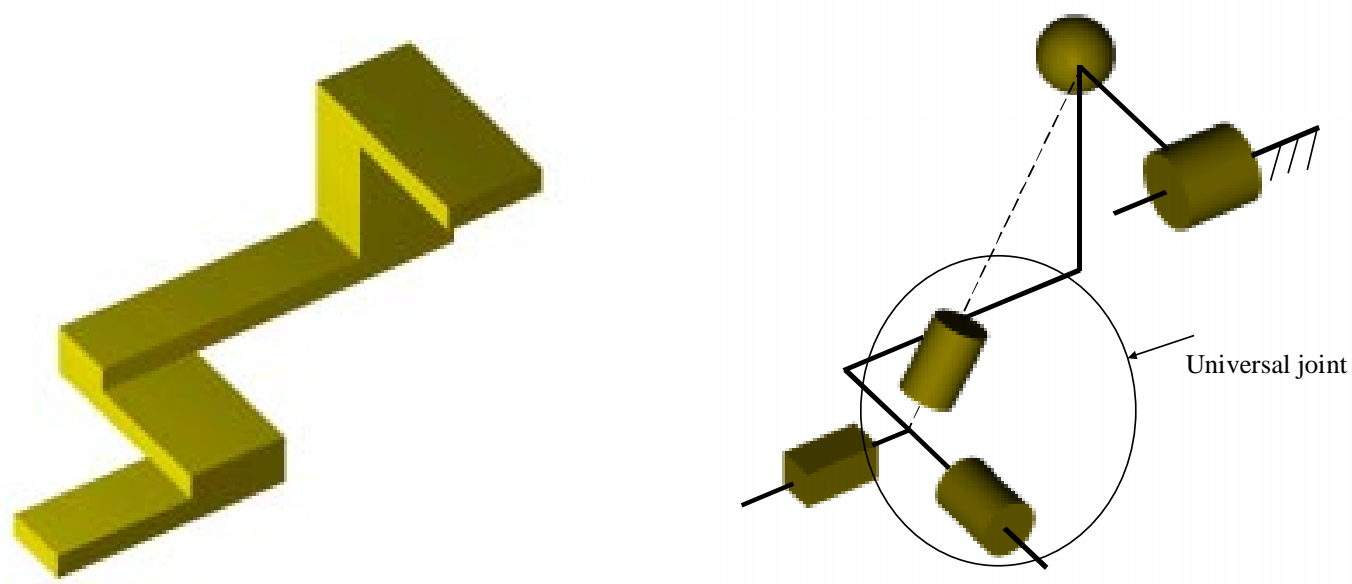

Fig. 13 (a) A mechanical part (b) After conversion to a mechanism

The tree graph of this mechanism represents a pair of links that are disconnected after cutting a joint. For a given initial estimate that does not satisfy the constraint function $\boldsymbol{\Phi}$, the set of linear equations is simultaneously solved for a correction $\delta \mathbf{q}^{(\mathrm{k})}$. The spherical joint is cut (because of the number of degrees of freedom), and the Jacobian matrix $\boldsymbol{\Phi}_{\mathbf{z}}^{\text {sph }}$ in Cartesian space can be transformed to joint space using velocity transformations as

$$
\boldsymbol{\Phi}_{\mathbf{q}}^{s p h}=\left[\boldsymbol{\Phi}_{\mathbf{z}}^{s p h} \mathbf{B}_{\text {rev }}\left|\boldsymbol{\Phi}_{\overline{\mathbf{z}}}^{s p h} \mathbf{B}_{\text {trans }}\right| \boldsymbol{\Phi}_{\mathbf{z}}^{s p h} \mathbf{B}_{\text {uni }}\right]
$$

where $\mathbf{B}_{\text {rev }}$ is the transformation matrix for the revolute joint $\mathbf{B}_{r e v}=\left[\begin{array}{c}\widetilde{\mathbf{r}}_{1} \mathbf{h}_{01} \\ \mathbf{h}_{01}\end{array}\right]$ and the axis is $\mathbf{h}_{01}=\mathbf{A}_{0} \mathbf{C}_{01}\left[\begin{array}{lll}0 & 0 & 1\end{array}\right]^{T}$, and $\mathbf{B}_{\text {uni }}$ is for the universal joint

$$
\mathbf{B}_{\text {uni }}=\left[\begin{array}{cc}
\tilde{\mathbf{r}}_{2} \mathbf{h}_{32} & \tilde{\mathbf{r}}_{2} \mathbf{g}_{32} \\
\mathbf{h}_{32} & \mathbf{g}_{32}
\end{array}\right]
$$

where $\mathbf{h}_{32}=\mathbf{A}_{3} \mathbf{C}_{32}\left[\begin{array}{lll}0 & 0 & 1\end{array}\right]^{T}$ and $\mathbf{g}_{32}=\mathbf{A}_{3} \mathbf{C}_{32} \mathbf{A}_{321}^{\prime \prime}\left[\begin{array}{lll}0 & 0 & 1\end{array}\right]^{T}$, where the rotation matrix is

$$
\mathbf{A}_{321}^{\prime \prime}\left(q_{2}\right)=\left[\begin{array}{ccc}
\cos \left(q_{2}\right) & -\sin \left(q_{2}\right) & 0 \\
\sin \left(q_{2}\right) & \cos \left(q_{2}\right) & 0 \\
0 & 0 & 1
\end{array}\right]
$$

For the translational joint, the velocity transformation matrix is $\mathbf{B}_{\text {trans }}$ defined as $\mathbf{B}_{\text {trans }}=\left[\begin{array}{ll}\mathbf{g}_{03} & \mathbf{0}\end{array}\right]^{T}$ and $\mathbf{g}_{01}=\mathbf{A}_{0} \mathbf{C}_{03}\left[\begin{array}{lll}0 & 0 & 1\end{array}\right]^{T}$, where

$$
\begin{array}{cc} 
& \boldsymbol{\Phi}_{\tilde{\mathbf{z}}_{i}}^{s p h}=\left[\begin{array}{ll}
-\mathbf{I} & \widetilde{\mathbf{r}}_{1}+\widetilde{\mathbf{s}}_{12}
\end{array}\right] \\
\text { and } & \boldsymbol{\Phi}_{\mathbf{z}_{j}}^{s p h}=\left[\begin{array}{ll}
-\mathbf{I} & -\left(\widetilde{\mathbf{r}}_{2}+\widetilde{\mathbf{s}}_{21}\right)
\end{array}\right]
\end{array}
$$

The initial estimates of the position and orientations of the ground are $\mathrm{x}=0, \mathrm{y}=0, \mathrm{z}=0, e_{1}=0$, $e_{2}=0$, and $e_{3}=0$. Initial estimates of joint attachment vectors in the body reference frame are presented in Table 2.

TABLE 2

Joint Attachment vectors in Body Reference Frame Estimates

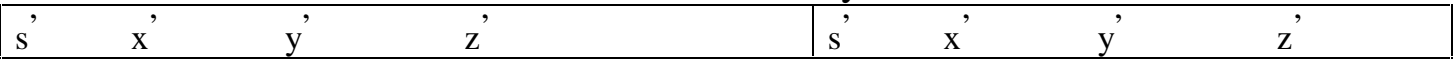




\begin{tabular}{|llll|llll|}
\hline $\mathbf{S}_{01}^{\prime}$ & 0 & 0 & 0 & $\mathbf{S}_{03}^{\prime}$ & 0 & 3.8637 & 0 \\
$\mathbf{S}_{12}^{\prime}$ & 2 & 0 & 0 & $\mathbf{S}_{32}$ & 0 & 0 & 0 \\
$\mathbf{S}_{21}^{\prime}$ & -2.828 & 0 & 0 & & & & \\
\hline
\end{tabular}

Transformation Matrices from Joint Coordinates to the Body Reference Frame are as follows.
$\mathbf{C}_{01}=\left[\begin{array}{lll}0 & 0 & 1 \\ 1 & 0 & 0 \\ 0 & 1 & 0\end{array}\right]$
$\mathbf{C}_{03}=\left[\begin{array}{lll}0 & 1 & 0 \\ 0 & 0 & 1 \\ 1 & 0 & 0\end{array}\right]$
$\mathbf{C}_{32}=\left[\begin{array}{lll}0 & 1 & 0 \\ 0 & 0 & 1 \\ 1 & 0 & 0\end{array}\right]$

Estimates of generalized coordinates are $\mathbf{q}=\left[\begin{array}{llll}45^{\circ} & -30^{\circ} & 0^{\circ} & 0^{\circ}\end{array}\right]^{T}$.

For a given initial estimate, the iterative method is used until the constraint violations are satisfied. A linkage length or a transformation matrix from joint coordinate to body reference frame may be changed. Therefore, joint attachment vectors may be changed (e.g., $\mathbf{s}_{o l}$, the revolute joint attachment vector in ground reference frame.) Once the linkage length has been changed, the constraint function is violated, and three options can be performed:

(1) The generalized coordinates $\mathbf{q}$ are changed such that

$$
\boldsymbol{\Phi}_{\mathbf{\mathbf { z }}_{1}}^{s p h} \mathbf{B}_{r e v} \delta q_{1}+\boldsymbol{\Phi}_{\mathbf{\mathbf { z }}_{2}}^{s p h} \mathbf{B}_{\text {trans }} \delta q_{4}+\boldsymbol{\Phi}_{\mathbf{\mathbf { z }}_{2}}^{s p h} \mathbf{B}_{\text {uni }}\left[\begin{array}{l}
\delta q_{2} \\
\delta q_{3}
\end{array}\right]=-\boldsymbol{\Phi}
$$

(2) The linkage lengths $\mathbf{s}^{\prime}$ are changed such that

$$
\begin{array}{ll}
\boldsymbol{\Phi}_{\hat{\mathbf{z}}_{1}}^{s p h} \mathbf{M}_{0} \delta \mathbf{s}_{01}^{\prime}+\boldsymbol{\Phi}_{\hat{\mathbf{z}}_{2}}^{s p h} \mathbf{M}_{0} \delta \mathbf{s}_{03}^{\prime}+\boldsymbol{\Phi}_{\hat{\mathbf{z}}_{2}}^{s p h} \mathbf{M}_{3} \delta \mathbf{s}_{32}^{\prime}+\boldsymbol{\Phi}_{\mathbf{s}_{l 2}} & \delta \mathbf{s}_{l 2}^{\prime}+\boldsymbol{\Phi}_{\mathbf{s}_{2 l}} \delta \mathbf{s}_{2 l}^{\prime}=-\boldsymbol{\Phi} \\
\text { where } & \mathbf{M}_{0}=\left[\begin{array}{c}
\mathbf{A}_{0} \\
\mathbf{0}
\end{array}\right] \quad \mathbf{M}_{3}=\left[\begin{array}{c}
\mathbf{A}_{3} \\
\mathbf{0}
\end{array}\right]
\end{array}
$$

$\boldsymbol{\Phi}_{\mathbf{s}_{12}}$ and $\boldsymbol{\Phi}_{\mathbf{s}_{21}}$ are spherical constraints.

(3) The generalized coordinates $\mathbf{q}$ and linkage lengths $\mathbf{s}$ are changed such that

$$
\begin{aligned}
& \boldsymbol{\Phi}_{\mathbf{\mathbf { z }}_{l}}^{s p h} \mathbf{B}_{r e v} \delta q_{1}+\boldsymbol{\Phi}_{\hat{\mathbf{z}}_{l}}^{s p h} \mathbf{M}_{0} \delta \mathbf{s}_{0 l}^{\prime}+\boldsymbol{\Phi}_{\hat{\mathbf{z}}_{2}}^{s p h} \mathbf{B}_{\text {trans }} \delta q_{4}+\boldsymbol{\Phi}_{\hat{\mathbf{z}}_{2}}^{s p h} \mathbf{B}_{u n i}\left[\begin{array}{c}
\delta q_{2} \\
\delta q_{3}
\end{array}\right]+\boldsymbol{\Phi}_{\mathbf{z}_{2}}^{s p h} \mathbf{M}_{0} \delta \mathbf{s}_{03}^{\prime} \\
& +\boldsymbol{\Phi}_{\mathbf{z}_{2}}^{s p h} \mathbf{M}_{3} \delta \mathbf{s}_{32}^{\prime}+\boldsymbol{\Phi}_{\mathbf{s}_{12}}^{\prime} \delta \mathbf{s}_{12}^{\prime}+\boldsymbol{\Phi}_{\mathbf{s}_{2 l}} \quad \delta \mathbf{s}_{2 l}^{\prime}=-\boldsymbol{\Phi}
\end{aligned}
$$

Initial estimates are provided for an assembled configuration. The design change due to adjusting the spherical joint attachment vector in crank reference frame $\mathbf{s}_{21}$ from $\left[\begin{array}{lll}-2.828 & 0 & 1\end{array}\right]^{T}$ to $\left[\begin{array}{lll}-2.8 & 0.2 & 0.1\end{array}\right]^{T}$ are illustrated in Fig. 14. 

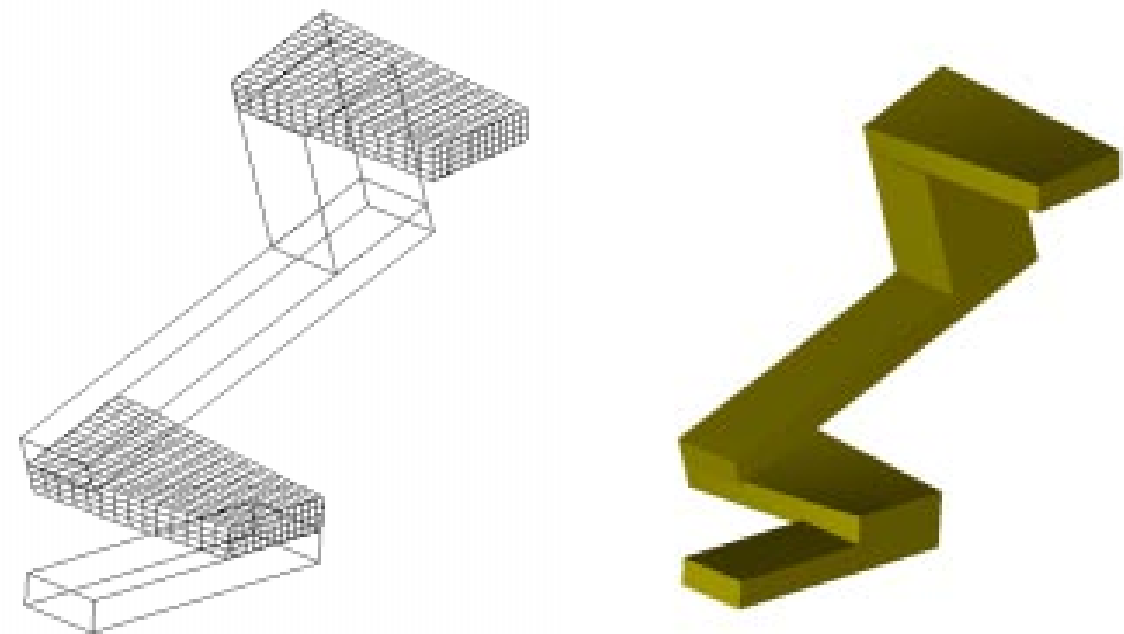

Fig. 14 The updated part after changes in the geometry are computed

\section{CONCLUSIONS}

A broadly applicable formulation for automating the parametrization and redesign of mechanical part geometry was presented. The iterative process typically associated with mechanical design is reduced by the use of such kinematically driven automated methods. Mathematical formulations based on earlier work are further expanded in this paper and are demonstrated to the automated computer-aided design of $2 \mathrm{D}$ and $3 \mathrm{D}$ mechanical parts. The significance of this formulation is evidenced in that it characterizes an alternative efficient method to the common parametric methods currently used. The main difference is the inherent kinematic formulation capable of computing admissible solutions very efficiently.

It was shown that mechanical parts represented in a CAD environment could be modeled into a mechanism comprising joints and links. It was shown that the deformation of the solid sustained by introducing a design variation is computed using a kinematic model. It was also shown that design changes through the mechanism are propagated such that an admissible configuration is computed using an efficient iterative generalized inverse method. The emphasis on masking this formulation from the CAD user (in an automated mode) is highlighted because of the long-term ramifications implied herein. The use of the inherent mechanism kinematics in modeling and providing solutions to the design assembly and parametrization scheme are automated in nature and are the focus of current research to expand this method to more complex shapes.

While the experimental computer code used to implement the algorithm is not refined, it has nevertheless demonstrated a new type of automatic design well suited for geometric parametrization of mechanical parts.

\section{ACKNOWLEDGMENTS}

This research was funded by the United States Army Tank-Automotive and Armaments Command (TARDEC) under the Automotive Research Center contract number DAAE-07-94C-R094. 


\section{REFERENCES}

1. Imamura, S, 1994, "Development of constraint based object oriented language for engineering design-on the parametric design support", Journal of the Japan Society for Precision Engineering 60(9):1242-1246.

2. Burke, E K, Elliman, D G, and Heard, M I, 1994, "XCODAMS: an engineering design system based on constraint propagation", Applications of Artificial Intelligence in Engineering, pp. 601-608

3. Cutkosky, M R and Tenenbaum, J M, 1990, "Toward a computational framework for concurrent engineering" Electronics Society-IECON, Pacific Grove, CA, pp700-706.

4. McMahon, CA; Cox, DR; Sims Williams, JH; Scott, JA, 1997, "Representation and Reasoning in Computer Aided Process Planning," Proceedings of the Institution of Mechanical Engineers, Part B: Journal of Engineering Manufacture, 211(B6):473-485.

5. Bowen, J and Bahler, D, 1992, "Frames, quantification, perspectives, and negotiation in constraint networks for life-cycle engineering" Artificial Intelligence in Engineering, 7: 199-226.

6. Fohn, SM, Greef, AR, Young, RE, and O'Grady, PJ, 1994, "Constraint system shell to support engineering approaches to concurrent engineering" Artificial Intelligence in Engineering, 9(1):1-17.

7. Kolb, M A and Bailey, M W, 1993, "FRODO: constraint-based object modeling for preliminary design" Proceedings of the 19th Annual ASME Design Automation Conference, Albuquerque, NM, 65:307-318.

8. Serrano, D and Gossard, D, 1988, "Constraint management in MCAE" Artificial Intelligence in Engineering Design (Gero, J S, editor), pp217-239.

9. Kott, A, Agin, G, Fawcett, D, 1992, "Configuration tree solver. A technology for automated design and configuration" Journal of Mechanical Design - Transactions of the ASME, 114(1):187-195.

10. Agrawal, R, Kinzel, GL, Srinivasan, R, Ishii, K, 1993, "Engineering constraint management based on an occurrence matrix approach", Journal of Mechanical Design, Transactions Of the ASME, 115(1):103-109.

11. Steinberg, L, Langrana, N, 1996, "EVEXED and MEET for mechanical design: testing structural decomposition and constraint propagation", Artificial Intelligence, 84(1-2) 37-56.

12. Jaques, MWS, Billingsley, J, Harrison, D, 1991 "Generative feature-based design-byconstraints as a means of integration within the manufacturing industry", Computer-Aided Engineering Journal, 8(6):261-267.

13. Abdel-Malek, K and Maropis, N, 1998 "Design-to-Manufacture Case Study: Automatic Design of Post-Fabrication Mechanisms for Tubular Components," SME Journal of Manufacturing Systems, 17(3):183-195.

14. Zou, HL, Abdel-Malek, K, and Wang, JY, 1996, "Computer-Aided-Design Using the Method of Cut-Joint Kinematic Constraints, Computer-Aided Design, 28(10):795-806.

15. Haug, E. J., 1989, Computer Aided Kinematics and Dynamics of Mechanical Systems, Vol I: Basic Methods, Allyn \& Bacon, Boston.

16. Zou, HL Abdel-Malek, K and Wang, J, 1997, Design Propagation in Mechanical Systems: Kinematic Analysis, ASME Journal of Mechanical Design, 119(3):338-345.

17. Abdel-Malek, K and Yeh, HJ, 1997, "On the Determination of Starting Points for Parametric Surface Intersections," Computer-Aided Design, 29(1):21-35. 